\title{
14 Addiction and the Diagnostic Criteria for Pathological Gambling
}

\author{
Neil Manson
}

\section{Introduction}

A philosophical question divides the field of addiction research. Can a psychological disorder count as an addiction absent a common underlying physical basis (neurological or genetic) for every case of the disorder in the category? Or is it appropriate to categorize a disorder as an addiction if the symptoms of and diagnostic criteria for it are sufficiently similar to those of other disorders also classified as addictions-regardless of whether there is some underlying physical basis common to each case of the disorder? The question concerns the scope and validity of the scientific concept of addiction and, more broadly, what is required for a psychological concept to count as scientific.

The case of pathological gambling (PG) raises this question nicely. "Should pathological gambling be considered an addiction?" asks Howard Shaffer (2003, 176). He specifies the question further (2003, 177-178): "When clinicians and scientists identify a behavior pattern as an addiction, even if they can identify it reliably according to DSM criteria, how do they know that it is indeed an addiction?" He warns that, as it stands, "the concept of addiction represents a troublesome tautology" $(2003,178)$ : a subject $S$ is addicted if and only if $S$ engages in repetitive behavior with negative consequences against $S$ 's better judgment. The problem with this concept is that it provides no way to distinguish behavior that cannot be controlled from behavior that is merely in fact not controlled. This "lay" concept is of little scientific value, Shaffer $(2003,179)$ argues, saying that "for addiction to emerge as a viable scientific construct ... investigators need to establish a 'gold standard' against which the presence or absence of the disorder can be judged." PG has no such gold standard, says Shaffer. What would such a gold standard be? Shaffer suggests "neurogenetic or biobehavioral attributes" (ibid.). Such attributes will have to be identified because "if pathological gambling represents a primary disorder orthogonal both to its consequences and the laws of probability, then clinicians and scientists should be able to identify the disorder without knowing the winning or losing status of the gambler" (ibid.). 
Stanton Peele $(2003,210)$ denies that there must be such a gold standard, claiming that researchers like Shaffer "fall prey to the reductionist fallacy" of thinking that pathological gambling must be fitted to "the disease model" if it is to count as an addiction.

This logic is exactly backwards-with drugs and with gambling. If a model does not begin to explain the behavior in question, then any number of associations with biological mechanisms and measurements will fail to provide an explanation (and, by extension, a solution) for the problem. Science is the creation of accurate and predictive models, not an exercise in laboratory virtuosity to show, for example, how drugs impact neurochemical systems. No work of this kind will ever explain the most basic elements of addiction, including that many people show the ability to be addicted to an array of dissimilar substances and activities, that different people react differently to the same addictive activities and substances even though the same neurological systems are implicated for all of these individuals, and-most patently—that people addicted to an involvement at one time and place cease to be addicted at a different time and place. (Ibid.)

In response to perceived encroachments from neurochemical reductionists, Peele $(2003,212)$ defends the autonomy of phenomenological psychology, claiming that "addiction is best understood from an experiential and behavioral perspective."

Shaffer $(2003,179)$ is right when he says clinicians should be able to identify PG "without knowing the winning or losing status of the gambler." However, as they currently stand, the accepted diagnostic criteria for PG fail to provide the desired ability. They rely on tacitly considering the fact that the gambling subject has actually suffered significant monetary losses. Because the obtaining of this fact is partially extrinsic to the subject (since gambling outcomes are partially a matter of chance), meeting the current diagnostic criteria for PG is neither necessary nor sufficient for being a pathological gambler. The criteria allow gambling outcomes to be determinative of PG when it should be the decisions gamblers make that play this role. The solution to this problem is to revise the screening instruments for PG so that they do not rely on knowledge of gambling outcomes. Clinicians need new diagnostic tools that are much more discriminating regarding the gambles being taken by the subjects they diagnose. Some new metrics for gambling quality are proposed, including metrics with a normative element.

\section{Problems with the DSM-IV criteria for PG}

The American Psychiatric Association's Diagnostic and Statistical Manual of Mental Disorders proposes that meeting five or more of the following diagnostic criteria is suffcient for classifying someone as a pathological gambler (assuming that "the gambling behavior is not better accounted for by a manic episode.")

(1) Preoccupation Subject is preoccupied with gambling (e.g., preoccupied with reliving past gambling experiences, handicapping or planning the next venture, or thinking of ways to get money with which to gamble) 
(2) Tolerance Subject needs to gamble with increasing amounts of money in order to achieve the desired excitement

(3) Withdrawal Subject is restless or irritable when attempting to cut down or stop gambling

(4) Escape Subject gambles as a way of escaping from problems or relieving dysphoric mood (e.g., feelings of helplessness, guilt, anxiety, depression)

(5) Chasing After losing money gambling, subject often returns another day to get even ("chasing" one's losses)

(6) Lying Subject lies to family members, therapist, or others to conceal the extent of involvement with gambling

(7) Loss of Control Subject has repeated unsuccessful efforts to control, cut back, or stop gambling

(8) Illegal Acts Subject has committed illegal acts such as forgery, fraud, theft, or embezzlement to finance gambling

(9) Risking Significant Relationships Subject has jeopardized or lost a significant relationship, job, or educational or career opportunity because of gambling

(10) Bailout Subject relies on others to provide money to relieve a desperate financial situation caused by gambling

(American Psychiatric Association 1994, 615)

Intuitively, meeting five or more of the preceding descriptions is an indication that a subject has a gambling problem. A closer look, however, reveals that at least one of the criteria-(9), "Risking Significant Relationships"-is dubious insofar as it depends on factors partially extrinsic to the subject. If it really is a valid criterion, then a community, or even just a single person, could make a pathological gambler out of a subject meeting four of the above criteria just by breaking off relationships with that subject. Whether or not someone is a pathological gambler could depend, say, on whether he or she lives in a conservative community that shuns gambling on religious grounds. This criticism has been made by Wakefield (1997), who notes that criterion (9) is "inconsistent with DSM IV's own definitions of mental disorder which assert that symptoms must not be due to conflict with society." It is hard to see how there could be a Shafferian physical "gold standard" connecting PG with addiction if PG itself cannot be diagnosed without reference to the subject's community.

These reflections on the problem with criterion (9) suggest that if we seek a Shafferian "gold standard" for addiction, then a general restriction must be enforced on diagnostic criteria for any proposed addictive behavior: the criteria must not make reference to factors extrinsic to the subject. What is it for a factor to be "extrinsic to the subject"? The general answer to this question is far from clear, yet that does not mean there are no particular factors we can declare extrinsic. It is an open question whether a subject's being accepted or rejected by her community reflects the state of the subject more than the state of the community. Clearly, however, whether three cherries do or do not come up on a slot machine has nothing to do with the subject, and the idea that a subject's having a psychological disorder might depend on whether three cherries come up is simply bizarre. 
The outcomes of the events wagered on by a gambler are at least partially outside of the gambler's control. Yet most of the DSM-IV criteria tacitly assume that the subject does, in fact, lose money on his or her gambles. That the DSM-IV criteria rely on this extrinsic consideration generates some glaring counterexamples to the claim that those criteria are adequate. Consider the following two cases.

(A) Mr. Lucky: The subject wins millions of dollars at a slot machine on his first visit to a casino. The subject subsequently becomes a frequent casino patron, playing slot machines exclusively and for long sessions. Due to the limits on the size of the wagers accepted by the slot machines, the subject is never in a position to risk a significant reduction is his overall slot machine winnings. After two years of frequent, intensive slotmachine play during which the subject suffers no significant monetary losses from his gambling, by chance he participates in a clinical study of gambling behavior and finds himself the subject of diagnosis.

(B) Mr. Unlucky: The subject is a brilliant young student of mathematics and psychology. After inheriting a significant amount of money, he decides to embark on a career as a professional poker player. He reads and fully grasps the best books on poker strategy, practices by playing low-stakes games online (with great success), receives tips and training from other professionals regarding how to read other players, learns to calculate the odds for all relevant poker situations in his head, and masters the art of keeping his "poker face." In short, he demonstrates all the requisite skills to be one of the world's best poker players. Finally, he moves to Las Vegas and begins playing poker for high stakes. Although he plays in a way that the other professionals regard as brilliant, he suffers an extremely long string of bad cards and "bad beats." After two years, all of his inheritance has been lost at the poker tables. He goes back to his parents, penniless and in need of their financial support. While home, the subject by chance participates in a clinical study of gambling behavior and finds himself the subject of diagnosis.

How would clinical psychiatrists, armed with the DSM-IV criteria for PG, diagnose our two hypothetical subjects?

Mr. Lucky almost certainly would not regard himself as a pathological gambler, and would not have sought treatment if he had not by chance participated in a clinical study of gambling behavior. But if he somehow found himself under the clinician's scrutiny, he would not be classified as a pathological gambler if that clinician relied solely on the DSM-IV criteria for PG. He does not exhibit withdrawal (3) because he never attempts to cut down or stop his gambling. It is not true that he gambles to escape his problems (4), seeing as his millions of dollars have alleviated whatever problems bothered him. He does not chase (5) insofar as there are no overall losses for him to chase; he is ahead millions of dollars. Rather than lying (6), the subject makes his gambling public; indeed, he brags about it as a way of attracting admirers and 
hangers-on. He never exhibits loss of control (7) because he has never had any reason to cut back or stop his gambling; after all, gambling made him a millionaire. He has never committed illegal acts (8) or requested bailout (10) because he has never needed to do so.

Despite all of this, intuitively Mr. Lucky has a serious gambling problem. Intuitively, no psychologically healthy person, when gifted with a prize of millions of dollars, would use it to spend his waking life playing slot machines-even if doing so never risked a significant loss of his money. Intuitively, there is something wrong with playing slot machines all day, even if doing so happens not to cause any financial hardship for the slot machine player. Mr. Lucky, it seems, is a pathological gambler if ever there was one-yet he would not be classified as such according to the DSM-IV criteria for PG. Thus meeting the DSM-IV criteria for PG is not required for someone to be a pathological gambler.

Meeting the DSM-IV criteria for PG is also not enough for someone to be a pathological gambler, as the case of Mr. Unlucky shows. If, as we have supposed, Mr. Unlucky is a brilliant poker player, then he is rightly preoccupied (1) with playing poker. Assuming that he gets a normal distribution of cards in his playing career, he rationally expects to make a significant amount of money in the long run by playing poker. In connection with this, he exhibits restlessness or irritability (3) if he is prevented from playing poker; after all, the less often he plays, the less money he can expect to make. Playing poker is his job, so not being able to do his job makes him restless and irritable. Whenever he loses, he comes back to play again (5). Given that he is a brilliant poker player, playing as much poker as he can is the smart thing for him to do, whether he has won or lost the day before. Because he seems to be cursed with ill luck, his friends abandon him (9), even though it is arguably irrational on the part of his friends to do so. (Can a subject be made a pathological gambler just by being abandoned by his friends-friends who believe in jinxes and curses?) Lastly, the subject is reduced to begging his parents for money (10).

Despite all of this, intuitively Mr. Unlucky is not a pathological gambler-or, if he is, then by parity of reasoning, there should also exist the categories "pathological investing," "pathological entrepreneurship," and so on. Yet clearly these fictional pathologies are bogus, unless we decide that the mere act of engaging in activities having high financial risks is pathological. Incidentally, the truncated life story of Mr. Unlucky, though fictional, mirrors that of known poker professionals, some of whom testify that early in their careers they started out with runs of extremely bad luck. These professionals testify that, though plagued by doubt, they continued to believe in themselves, and eventually their fortunes turned. We can say of these professionals that they did the right thing in continuing to play despite their losses. This is because skill at poker is an objective property of a person-at least, just as much an objective property as skill at investing, skill at starting up a business, and so on. The skilled business 
owner whose first enterprise fails through misfortune clearly does the right thing by trying to start up another business. Indeed, a skilled business owner does the right thing in trying to start up another business no matter how many prior businesses have failed.

Of course, the failure of many business ventures is good evidence that the business owner is not, in fact, skilled, but rather is inept. But this epistemic point is irrelevant here. Supposing that the business owner really is skilled, bad luck with past business ventures is no reason to abandon future ventures (and if the APA cared to identify a new disorder, "pathological entrepreneurship," they would have to find a way of identifying it that did not depend on knowing whether a subject's businesses succeeded or failed). Likewise, supposing Mr. Unlucky really does have all of the skills necessary to be one of the world's greatest poker players, initial bad luck is no reason for him to stop playing poker. A fortiori it is no reason to classify him as a pathological gambler.

Note that a person need not know or be confident that he possesses an ability to possess that ability. Some teachers are familiar with the phenomenon of students insisting they just do not understand certain material, even though all objective tests show that the students have mastered that material. ${ }^{1}$ Suppose Mr. Unlucky is likewise plagued with doubt about his skill. He might mistakenly attempt to cut down on his poker playing, yet fail to do so, thus exhibiting loss of control (7). He might also wrongly feel ashamed of his losses, attributing them to lack of skill rather than bad luck, and thus might lie about his gambling (8). In this case, Mr. Unlucky would meet seven out of the ten criteria for PG. Nonetheless, intuitively Mr. Unlucky is not a pathological gambler. He has simply (i) started off with an exceptionally bad run of luck, and (ii) mistakenly attributed his losses to himself rather than to the bad run of luck.

The case of Mr. Unlucky shows that meeting the DSM-IV criteria for PG is not sufficient for someone to be a pathological gambler; that is, it does not guarantee that someone is a pathological gambler. The case of Mr. Lucky shows that meeting those criteria is not necessary for someone to be a pathological gambler. Thus meeting the DSM-IV criteria for PG is neither necessary nor sufficient for being a pathological gambler. Meeting the criteria does not entail that the subject is a pathological gambler, and not meeting the criteria does not entail that the subject is not a pathological gambler. The source of the problem is that the APA has tacitly made the status "having lost significant money" - a status partially extrinsic to the gambler-be criterial of PG.

What is the practical import of this problem with the DSM-IV criteria for PG? As Stinchfield, Govoni, and Frisch $(2007,205)$ note, the DSM-IV criteria are the standard criteria in PG research:

In response to the need for instruments to detect and measure problem gambling, a number of instruments have been developed. The SOGS is the most commonly used assessment instrument and has accumulated the largest volume of psychometric research to date, but new DSM-based instruments are generating a good deal of research momentum. DSM-IV diagnostic criteria have 
been paraphrased into questions that are used to diagnose clients in gambling treatment programs (e.g., DIGS), measure prevalence rates of PG in epidemiological surveys (e.g., DSM-IV-MR, NODS, and GAM), and measure gambling treatment outcomes (e.g. GAMTOMS).

Petry's $(2005,35-54)$ survey of PG screening instruments comports with that of Stinchfield, Govoni, and Frisch. Except for SOGS-the South Oaks Gambling Screen (Lesieur and Blume 1987), which was informed by DSM III-every screening instrument mentioned by Petry was either modified or developed directly in light of the DSM-IV criteria for PG. If the DSM-IV criteria for PG are fundamentally inadequate (as argued above), then any search for a gold standard for PG based on those criteria is bound to fail. The question that arises is how the DSM-IV criteria might be amended or supplemented to put the classification on a more solid footing.

\section{Developing a Metric of Gambling Quality}

The problem with the DSM-IV criteria seems to have arisen due to ignorance on the part of the psychiatric and psychological communities regarding some relevant specifics of gambling. They have treated gambling as a blob. They have used the term "gambling" as a mass noun and seen all forms of it as essentially alike. The following characterization of gambling is typical:

It is well accepted that statistical principles of probability applied to gambling indicate that payout rates and overall advantage always favor the house. The cost of each gamble is a combination of the proportion of each bet retained for taxes and the "house edge," with the remainder allocated to a prize pool for distribution amongst winners. This means that the longer one gambles, the greater the likelihood of losing. Under these conditions, an economically prudent person would minimize risk by limiting exposure to gambling. (Blaszczynski and Nower 2007, 323-324)

Yet gambling is not a blob; there is a tremendous variety of events on which a person may stake a wager. The events wagered on and the kinds of wagers made defy reduction to the sorts of easy generalizations made above. First, not all gambling involves competition against "the house," as suggested by the above quotation. For example, poker is played against other players, with the house charging a fee, either in the form of taking a percentage of each pot or in the form of an explicit entry fee for poker tournaments. Second, it is not true that "all gambling outcomes are determined by chance," if this is taken as meaning skill has no role in gambling; again, the case of poker illustrates this. The false generalization that gambling is "all a matter of chance" helps explain the befuddlement of some researchers at the fact that "gamblers rated their peers who won as more competent than those who lost" (Blaszczynski and Nower 2007, 330). Although it is true for many forms of gambling that there is no such thing as skill with respect to them, some games do involve some level of skill. Depending on the type of game, winning might indeed be a sign of competence. Finally, although it 
is true that "payout rates and overall advantage always favor the house," games differ tremendously in their payout rates and the overall advantage they provide to the house (the "house edge"). Some games have a fast rate of play and the house edge on each wager is high; others have a slow rate of play and the house edge on each wager is relatively low. The concern shown by casinos for marketing specific games, and for placing specific games in specific areas of the casino, attests to these facts. The differences in rate of play and house edge are crucial in evaluating the gambling engaged in by the subjects in PG studies. Yes, most gamblers wager against the house, make wagers for which the house has a considerable edge, and exercise little or no skill when they gamble; but not all do. The DSM-IV criteria for PG are blind to this fact.

Instead of diagnostic criteria that tacitly refer to the losing status of the gambler, PG research needs criteria that incorporate a refined, multidimensional metric of the games being played-a metric that ties in directly to the comparative quality of the decisions made by the gambler. Some researchers have made efforts to develop general metrics of gambling behavior and gambling decisions, but these metrics neglect the nuts and bolts of the specific games on offer, both in casinos and elsewhere. For example, in "Quantification and Dimensionalization of Gambling Behavior," Currie and Casey $(1997,163)$ identify the dimensions of gambling activity as "participation status, frequency, expenditure, duration, and type of game played." Yet their account of the dimension "type of game played" (ibid., 168) is thin: "There are literally hundreds of different gambling activities, each with its own set of rules, odds of winning, and payout schedule," they note. "Gambling types can be categorized across several dimensions: single-player (e.g., blackjack) versus multiplayer (e.g., bingo); passive (e.g., sports betting) versus active (e.g., poker); continuous (e.g., slot machines) versus noncontinuous (e.g., lottery)." This list of distinctions provides a helpful start, but much more detail is needed.

A more refined metric should include at least the following dimensions: (a) the expected value of the wager in question; (b) the rate of play at which the wager in question is being made; (c) the degree to which the making of the wager and the observation of the outcome require social interaction; (d) the degree to which strategic thinking is required for the placement of the wager; and (e) the degree to which the wager involves competition against other agents. This list of dimensions is not put forth as exhaustive. Perhaps others need to be considered. But the ones offered here have two advantages. First, unlike the binary dimensions offered by Currie and Casey, each of these dimensions is scalar, so a metric based on them allows for more refined classifications than does that of Currie and Casey. Second, collectively they incorporate and supplant those suggested by Currie and Casey. "Rate of play" supplants "continuous versus non-continuous." "Social setting" supplants "single player versus multiplayer." "Strategic thinking" and "competition" supplant "passive versus active." Below I give more detailed descriptions of each of these proposed dimensions. 
(a) Expected value The concept of expected value is explained in connection with casino games by Manson (2003). We can simply note here that attached to each wager is a number representing the percentage of the dollar amount wagered that the gambler can expect to get in return "in the long run" - that is, the average return on each specific wager if that specific wager were to be made over and over again. This number is also sometimes represented as the "house edge," which is simply 100\% minus the expected value. Thus for roulette (with two zeros), every wager (but one) has an expected value of $94.7 \%$ and thus a house edge of $5.3 \%$. The "pass line" bet in craps has an expected value of $98.6 \%$; furthermore, making it entitles the bettor to place a true "odds" bet (a bet with an expected value of 100\%) of much greater value than the pass line bet. Thus the overall expected value of a pass line plus odds bet can get extremely close to $100 \%$ (if the bettor stakes a lot of money). Depending on one's strategy and on the rules of the house for it, the expected value for a hand of blackjack can approach $99 \%$. At the opposite extreme, in most state-run lotteries the expected value of a $\$ 1$ ticket is at or below $50 \notin$.

(b) Rate of play The rate of play refers to the rapidity with which the gambler makes each individual wager (say, one hand of blackjack per minute). Slot machines typically have the highest rate of play; gamblers can wager perhaps ten times in a minute on certain machines. For craps, the time between each roll of the dice can range anywhere from fifteen seconds (if the table consists of only a single player making a single bet) to several minutes (if the table is full and players are making a variety of bets). Likewise, the speed at which hands of blackjack are dealt or spins of the roulette wheel are made depends on the number of players at the table and the size and variety of their bets. Poker hands vary depending on these factors plus the time it takes players to make decisions. For lotteries and sports wagers, on the other hand, there is a significant gap in time-sometimes several days-between the placing of the wager and the occurrence of the event wagered on.

Several points need making here. First, typically the expected value and rate of play bear an inverse relationship to one another. The lower the house edge is, the more rapidly the game is played; the slower the game, the bigger the house edge. The dynamic is just like that in retail sales. Some businesses sell items at low marginal profit but make up for it with a high sales volume, whereas purveyors of luxury items sell their wares at large markups because luxury items are rarely bought.

Second, seemingly small differences in the expected values of two bets can lead to a very large difference in the overall expected returns of series of those bets. In craps, the expected value of a "proposition bet" on the number 12 is $86.1 \%$, whereas the expected value of a pass line bet is $98.6 \%$, as is the expected value of a "come" bet. Suppose two players go to a craps table, each with $\$ 1,000$. On every roll of the dice the one makes a $\$ 10$ proposition bet on 12 , while the other makes either a $\$ 10$ pass 
line bet or a $\$ 10$ "come" bet. Each plays craps for five straight hours. If the dice were tossed at a rate of once per minute, each player has placed $\$ 3,000$ in wagers. This is possible because it is not necessary to have $\$ 3,000$ to make $\$ 3,000$ worth of bets over the course of five hours-unless one loses every single bet, which is extremely unlikely. Mathematically, the first player would be expected to lose $\$ 425$, the second only $\$ 45$. The first player is expected to lose about $\$ 85$ per hour, the second about $\$ 8$ an hour. Arguably, the second player might count as the "economically prudent person" mentioned by Blaszczynski and Nower once the various perquisites of casino gambling (free drinks, entertainment, and so on) are considered. At the very least, the second player is engaged in a leisure activity with a per-hour cost comparable to those of other leisure activities (bowling, golf), with one exception: the per-hour cost of gambling is variable, whereas the per-hour cost of other leisure activities is fixed. Unlike other leisure activities, gambling involves risk with respect to cost.

Third, just as having a negative expected value does not necessarily make it irrational (not "economically prudent") to engage in a form of gambling, having a positive expected value does not necessarily make it rational to engage in a form of gambling. Consider a hypothetical game in which a slow-churning, robotically controlled machine mixes 4,999 red balls in an urn along with 5,001 white balls. Every ten minutes, a ball is drawn, its color is noted, and then it is placed back in the urn. The casino offers to pay even money to bettors who correctly predict the color of the ball drawn; the maximum bet is $\$ 10$. Given these rules, the player playing this game nonstop can be expected to make $\$ 10$ every seven days. The game involves no strategy and no competition (two dimensions of gambling to be discussed shortly), and it is intensely monotonous. Intuitively, anyone who did play this game repeatedly would be irrational, even though the gambler would be expected to make money playing it. This suggests that judging a form of gambling to be rationally engaged in or not involves a much more complex judgment than just that the form of gambling has a negative expected value. Additional dimensions besides expected value and rate of play must have some role. I suggest three such additional dimensions below.

(c) Social interaction Intuitively, any metric of gambling behavior that wants to detect PG should capture whether the subject's behavior involves significant social interaction. The dimension "single player versus multiplayer" of Currie and Casey indirectly gets at the relevant question: is the gambler engaged in his or her activity alone, or in the company of others? To divide the question more precisely, is the wager placed alone, and is the outcome observed alone? For some gambles (e.g., slot machines and lotteries), both the placement of the wager and the observation of the outcome typically occur alone. For others (e.g., sports bets), the wager is typically placed alone but the outcome is typically observed communally. For some games (e.g., roulette, craps, and blackjack), the placement of the wager and the observation of the outcome typically both occur in a crowd (though it is possible to find just a single player at a rou- 
lette, craps, or blackjack table). Other games (e.g., poker) necessarily involve the joint effort of gamblers. And for almost all of these games, the degree to which other people are involved varies. For example, one can play blackjack alone if one finds a table with no other players. Note that the degree of social interaction is typically related to the rate of play; the more people that are involved in a game, the longer it takes for the result of each particular wager to be determined.

(d) Need for strategy For some games, the decisions made by the gambler affect the expected value of the wager made. This is obviously the case in games such as poker, which involve such decisions at every stage. Bets on roulette wheels, on the other hand, involve no strategic decision making. But many games involve strategy to some degree. Blackjack is an obvious example; whether to "stay" or take a "hit" affects the probability that the player will win his or her wager. Knowing when to stay and when not to involves some knowledge; that is why there are various strategy "systems" for blackjack, spelled out clearly in pricey books and pamphlets. Even craps involves decisions regarding which bets to make, with the bets varying with respect to their expected values; to that extent, craps involves strategy, even if the outcomes of the dice rolls themselves are completely beyond human control.

Such decisions engage the intellect. Insofar as it is intrinsically better for an activity to engage the intellect than not, games that require such decisions are, other things being equal, better than games that do not. Strategic games reflect better on those who engage in them than do nonstrategic games. Other things being equal, it is better to play chess than tic-tac-toe. Likewise, other things being equal, it is better to play poker than slot machines. This value difference can be captured if we measure games along dimension $(\mathrm{d})$.

(e) Degree of competition Some games involve outthinking and outplaying another agent, or at least an artificial agent. Chess requires paying attention to one's opponent, figuring out her patterns, anticipating her next move, and so on. Chess thus engages the intellect in a way that few other games do, though the kinds of thinking involved with competing against another agent are distinct from those mentioned in the "strategy" dimension discussed above. Thus there is an element to playing chess against an opponent (real or virtual) that is missing from, say, roulette. Insofar as it is intrinsically better for an activity to engage the intellect than not, games that feature competition are, other things being equal, better than games that do not. Competitive games reflect better on those who engage in them than do noncompetitive games. The value difference between competitive and noncompetitive forms of gambling should be accounted for in any metric for game quality. This value difference can be captured if we measure games along dimension (e).

Intuitively, poker, which involves direct competition against other gamblers, is a form of gambling that speaks better of those engaging in it than slot machines. Aside 
from the varieties of poker, no other casino games involve competition. This assumes we do not view the casino itself as an agent, but rather as an automaton; this assumption is justified, because, unlike even a computerized opponent in chess, the house always follows a predesignated and preannounced strategy. However, some forms of noncasino gambling (e.g., sports wagering) do involve some degree of competition. The important point for researchers and clinicians is that the degree of competition a game features is relevant in assessing the person making the decision to play that game. That a form of gambling taps into human competitive instincts is surely just as much a factor in its attractiveness as the fact that it offers those playing it a chance to make money.

Indeed, the desire to compete is plausibly regarded as virtuous. In The Politics Aristotle proposed that a good political structure would discourage conflict but encourage competition (Skultety 2009). Aristotle held that competition is intrinsically good because it promotes excellence, and that it is derivatively good because it develops virtues such as courage. Plausibly, the development of courage requires the suppression of riskaversion instincts. Although some forms of gambling (e.g., slot machines) may desensitize gamblers to risk in the wrong way (by encouraging impulsive action), others (e.g., poker) may do so in the right way (by encouraging strategic thinking and deliberation). Undoubtedly, few of the gambling activities people engage in actually do develop virtues such as bravery or judgment, and the absence of those virtuous traits from most forms of gambling is doubtless part of what makes subjects who routinely engage in those forms of gambling disordered. To repeat, the important point for researchers is that what might contribute to making a form of gambling bad is that it involves no competition, or that it involves a degenerate form of competition-not just that those engaging in it can be expected to lose money.

\section{Conclusion}

The import of the preceding discussion should be clear. There is a strong cognitive component to gambling behavior. It is the cognitive features of gambling decisions that PG researchers need to measure, not the outcomes of those decisions, if we ever hope to find a gold standard for PG. Yes, significant gambling losses correlate strongly with a subject's being a bad decision maker with respect to gambling, but that is no excuse for making gambling losses criterial of PG. Doing so makes factors extrinsic to the subject be determinative of PG. This would rule out the possibility of a gold standard for PG and cast grave doubt on whether PG could properly be considered an addiction.

Note

1. As a long-time logic teacher, I have known students who insisted they did not know how to complete proofs in symbolic logic - yet they successfully completed all of their assigned proofs. 
Their success was a mystery to them. One student occupied my office hours trying to get me to explain where he was going wrong in his proofs. When I told him his proofs were flawless (just as all his prior work had been), he was incredulous.

\section{References}

American Psychiatric Association (1994). Diagnostic and Statistical Manual of Mental Disorders, 4th edition. Washington, D.C.: American Psychiatric Association.

Blaszczynski, A., and Nower, L. (2007). Research and measurement issues in gambling studies: Etiological models. In Garry Smith, David C. Hodgins, and Robert J. Williams, eds., Research and Measurement Issues in Gambling Studies, pp. 323-344. New York: Elsevier.

Currie, S. P.. and Casey, D. M. (1997). Quantification and dimensionalization of gambling behavior. In Garry Smith, David C. Hodgins, and Robert J. Williams, eds., Research and Measurement Issues in Gambling Studies, pp. 155-177. New York: Elsevier.

Lesieur, H. R., and Blume, S. B. (1987). The South Oaks Gambling Screen (SOGS): A new instrument for the identification of pathological gamblers. American Journal of Psychiatry 144 (9): 11841188 .

Manson, N. A. (2003). Probability on the casino floor. In Gerda Reith, ed., For Fun or Profit? The Controversies of the Expansion of Gambling, pp. 293-307. Amherst, N.Y.: Prometheus Books.

Peele, S. (2003). Is gambling an addiction like drug and alcohol addiction? Developing realistic and useful conceptions of compulsive gambling. In Gerda Reith, ed., For Fun or Profit? The Controversies of the Expansion of Gambling, pp. 208-218. Amherst, N.Y.: Prometheus Books.

Petry, N. M. (2005). Pathological Gambling: Etiology, Comorbidity, and Treatment. Washington, D.C.: American Psychological Association.

Shaffer, H. J. (2003). A critical view of pathological gambling and addiction: Comorbidity makes for syndromes and other strange bedfellows. In Gerda Reith, ed., For Fun or Profit? The Controversies of the Expansion of Gambling, pp. 175-190. Amherst, N.Y.: Prometheus Books.

Skultety, S. (2009). Competition in the best of cities: Agonism and Aristotle's politics. Political Theory 37: 44-68.

Stinchfield, R., Govani, R., and Frisch, G. R. (2007). A review of screening and assessment instruments for problem and pathological gambling. In Garry Smith, David C. Hodgins, and Robert J. Williams, eds., Research and Measurement Issues in Gambling Studies, pp. 179-213. New York: Elsevier.

Wakefield, J. S. (1997). Diagnosing DSM-IV, part 1: DSM-IV and the concept of disorder. Behavior Research and Therapy 36: 633-649. 
